REVISTA ECONOMÍA

Vol. 70, N. ${ }^{\circ}$ III (mayo 2018), 47-67

\title{
LA LEY GENERAL DE LA ACUMULACIÓN CAPITALISTA: UNA REINTERPRETACIÓN
}

\author{
JOHN CAJAS GUIJARRO \\ Universidad Central del Ecuador \\ Recepción de manuscrito: 28 de enero de 2018 \\ Aceptación versión final: 13 abril de 2018
}

\begin{abstract}
RESUMEN Este artículo reinterpreta uno de los pasajes más importantes de El Capital: la «ley general de la acumulación capitalista», en donde la contradicción asalariados-capitalistas genera una dinámica cíclica en la acumulación de capital. Para lograr tal reinterpretación -similar a la propuesta de Richard Goodwin (1967) — se definen los determinantes de la acumulación y la tasa de ganancia, se detallan las funciones del ejército laboral de reserva (ELR), y se juntan ambos aspectos en un sistema dinámico cuya simulación sugiere la existencia de ciclos capitalistas afectados por la tendencia a la caída de la tasa de ganancia.
\end{abstract}

PALABRAS CLAVE Ley general de la acumulación capitalista, ejército laboral de reserva, sistema dinámico, ciclos.

ABSTRACT This paper reinterprets one of the most important passages of Capital: the «general law of capitalist accumulation", where contradiction between workers and capitalists causes cyclical dynamics in capital accumulation. To achieve the reinterpretation - similar to Richard Goodwin (1967) proposal - the determinants of capital accumulation and the rate of profit are identified, it is presented a description of the reserve army of labour and its functions, and both aspects are joined in a dynamical system whose simulation suggests the existence of capitalist cycles influenced by the tendency of the rate of profit to fall.

KEYWORDS General law of capitalist accumulation, reserve army of labour, dynamical system, cycles.

JEL CODES C63, E11, O41.

\section{INTRODUCCIÓN}

El capital es un libro que ha generado grandes controversias, muchas veces protagonizadas por quienes nunca han leído más allá de sus primeros capítulos. A fin de enfrentar tal limitación, el presente artículo reinterpreta una de las conclusiones más importantes del tomo I de El capital de C. Marx, publicado el 14 de septiembre de 1867 . El pasaje a reinterpretarse no es ni la teoría del valor, ni la «transformación del dinero en capital», ni la teoría del plusvalor. ${ }^{2}$ El pasaje por reinterpretarse es uno de los capítulos finales del tomo I, titulado en español como la 
ley general de la acumulación capitalista (Marx, 1867, cap. XxiII). Según dicha «ley», se puede leer que en el capitalismo la contradicción asalariados-capitalistas — reflejada en la pugna salarios-ganancias - genera una posible dinámica cíclica del proceso de acumulación capitalista, matizada por la tendencia a la caída de la tasa de ganancia.

Cabe aclarar que el artículo no busca ser es un reflejo fiel de la palabra de Marx. Lo que realmente se busca, desde las intuiciones que Marx dejó en su «ley general», es armar una reinterpretación analítica que mejore el entendimiento - y genere mayores dudas- sobre la dinámica capitalista, en especial sobre su potencial comportamiento cíclico y el efecto que podría tener la tasa de ganancia en el ciclo. ${ }^{3} \mathrm{Si}$ bien las reinterpretaciones de la «ley general de la acumulación capitalista» son múltiples —destacando Goodwin (1967) —, sin embargo, no parecen aprovechar varias intuiciones que Marx dejó. En especial, no parece existir una interpretación clara de los determinantes de la acumulación, y el papel que cumple el «ejército laboral de reserva» (ELR) ${ }^{4}$ dentro de la acumulación — funciones de reserva y de explotación-. Tampoco parece existir un análisis claro del papel que cumpliría, en tal proceso, el vínculo entre tasa de ganancia y mecanización — aspecto que, por ejemplo, Goodwin no profundizó-.

Así, el objetivo de este artículo es reinterpretar la «ley general de la acumulación capitalista» planteada casi como conclusión del tomo I de El capital, pero aprovechando las intuiciones que Marx propuso sobre el ELR de forma más completa que en otras reinterpretaciones. Igualmente, se busca brindar bases para debates futuros sobre cómo interpretar la mencionada «ley» e incluso sobre el papel que dentro de ésta cumpliría la tasa de ganancia. Para cumplir esto, el artículo se compone de tres secciones. Después de esta introducción — sección 1- se reinterpreta la «ley general» — sección 2- en tres partes: primero se definen los determinantes de la acumulación capitalista y de la tasa de ganancia; después se detallan las funciones de reserva y de explotación del ELR —incluyendo un potencial efecto sobre precios-; posteriormente se juntan las partes en un sistema dinámico cuya simulación numérica identifica la posible existencia de ciclos capitalistas, resultado con el cual es posible replantear tanto la «ley general» como la «tendencia decreciente de la tasa de ganancia». Finalmente, se cierra con una conclusión - sección 3- en donde se destaca la importancia política de los resultados.

\section{REINTERPRETANDO LA LEY GENERAL DE LA ACUMULACIÓN CAPITALISTA}

\section{DETERMINANTES DE LA ACUMULACIÓN CAPITALISTA}

La dinámica básica del capitalismo consiste en usar un monto inicial de dinero (D) para comprar mercancías (M) divididas en medios de producción (MP) y fuerza de trabajo (FTR) las cuales, al interactuar en la producción (P), crean nuevas mercancías ( $\mathrm{M}^{\prime}$ ) cuya venta genera un monto de dinero (D') mayor al monto inicial gastado, en donde la diferencia genera una ganancia (G). Tal lógica se recoge en el ciclo de circulación del capital productivo (Marx, 1885, p. 57), representado en la expresión (o).

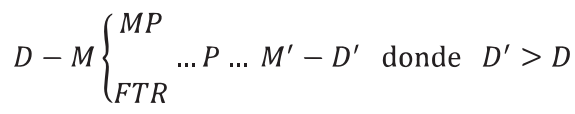


Al surgir la ganancia, el dinero inicial (D) se transforma en representante de un capital total (K) a la vez que sus dueños se transforman en capitalistas. Asimismo, el dinero gastado en medios de producción representa un capital constante (c) mientras que el dinero gastado en adquirir fuerza de trabajo se transforma en representante de un capital variable (v) que termina en manos de la clase asalariada —el cual se asumirá que se destina, por completo, al consumo- (Marx, 1867, pp. 250-253). Esta distribución del dinero que representa al capital se la representa en (1), (2) y (3).

$$
\begin{gathered}
K=c+V \\
C=p A \\
V=w h E
\end{gathered}
$$

Donde A es la cantidad de medios de producción empleados, ${ }^{5} \mathrm{p}$ es el precio de dichos medios - que se asumirá igual al de todas las demás mercancías - ${ }^{6} \mathrm{E}$ es el número de empleados que venden su fuerza de trabajo, h es el número de horas de trabajo por persona y w es el salario promedio nominal por hora de trabajo.

Sobre la ganancia, ésta puede definirse como la diferencia entre el ingreso obtenido al vender un determinado volumen de mercancías producidas $(\mathrm{Q})$ a un precio (p), descontando salarios (v) y descontando los gastos por degaste de medios de producción (donde $\mathrm{o}<\delta<1$ es una tasa de depreciación) aplicada al gasto en dichos medios (c).7 Tal definición de las ganancias se recoge en (4).

$$
G=p Q-(V+\delta C)
$$

A su vez la tasa media de ganancia (g) se define como la ratio entre las ganancias y el dinero gastado en capital constante y variable, definición recogida en (5).

$$
g=\frac{G}{K}
$$

Respecto al volumen de mercancías producidas, éste se expresa como el producto del número de personas empleadas, las horas de trabajo y la «productividad laboral» promedio por hora de trabajo, como indica (6).

$$
Q=q h E
$$

Aquí se considerará que la "productividad laboral» aumenta al crecer la intensidad del trabajo (e), ${ }^{8}$ al crecer la mecanización (m) - ratio entre medios de producción y personas empleadas-, o al cambiar otros factores (a10), como indican (7) y (8).

$$
\begin{gathered}
q=f_{1}\left(e, m, \alpha_{10}\right), f_{1 e}>0, f_{1 m}>0 \\
m=\frac{A}{E} \quad \text { (8) }
\end{gathered}
$$

Si, resultado de la producción capitalista, surge una ganancia y una tasa de ganancia positivas $(\mathrm{G}>\mathrm{o}, \mathrm{g}>\mathrm{O})$, entonces el dinero en manos de la clase capitalista crece, aumentando así su poder. ${ }^{10}$ Para que eso ocurra se necesita que la "productividad laboral» (q) supere a la suma entre el salario real (w/p) y los gastos de depreciación, como indica (4'). ${ }^{11}$

$$
G>0, g>0 \leftrightarrow q>\frac{w}{p}+\delta\left(\frac{m}{h}\right)
$$

Ahora, si se asume que el poder requiere de más poder para «sobrevivir», ${ }^{12}$ entonces, luego de 
obtener la ganancia, para garantizar su existencia la clase capitalista estaría obligada a destinar parte de ésta a ampliar el capital total y, así, obtener más ganancias y ampliar su poder -lógica exacerbada por aspectos como la competencia capitalista (Marx, 1867, p. 697, pp. 731-732) Así, surge la acumulación de capital, es decir, la ampliación del capital, financiada —en el caso más básico- ${ }^{13}$ con el ahorro de una fracción (s) de las ganancias capitalistas, esperando que tal ahorro se canalice - gracias al sistema financiero - hacia la compra de una mayor cantidad de medios de producción y fuerza de trabajo, ${ }^{14}$ descontando los gastos de pérdida de medios de producción causados por el desgaste. Por tanto, la acumulación de capital puede representarse como el cambio en el tiempo del dinero que representa al capital total, financiado por el ahorro de una fracción de las ganancias y descontando el gasto de depreciación aplicado al capital constante, ideas recogidas en (9). ${ }^{15}$

$$
K^{\prime}=s G-\delta C
$$

Donde $\mathrm{K}^{\prime}=\mathrm{d} \mathrm{K} / \mathrm{dt}$ es el cambio del dinero que representa al capital total, el cual se representa como una derivada temporal. ${ }^{16}$ También es posible definir la tasa de crecimiento de ese dinero, a la cual se denomina tasa de acumulación (y) y se representa en (10).

$$
\gamma_{K}=\frac{K^{\prime}}{K}
$$

Usando (1) a (10) se puede definir los determinantes de la tasa de acumulación y de la tasa de ganancia, los cuales se recogen en (ACU1) y (ACU2). ${ }^{17}$

$$
\begin{gathered}
\gamma_{K}=s g-\frac{\delta m}{m+h(w / p)} \quad(A C U 1) \\
g=\frac{h(q-w / p)-\delta m}{m+h(w / p)} \quad(\text { ACU2) }
\end{gathered}
$$

Donde $\mathrm{q}=\mathrm{f}_{1}\left(\mathrm{e}, \mathrm{m}, \mathrm{a}_{10}\right)$ y bajo la condición (4').

Revisando (ACU1) se nota que la tasa de acumulación crece si crecen las tasas de ahorro, de ganancia y/o el salario real, mientras que se contrae si crece la depreciación y/o la mecanización. Por su parte, (ACU2) muestra que la tasa de ganancia crece cuando crecen las horas trabajadas y/o la "productividad laboral», y cae cuando crece la depreciación, el salario real y, en especial, la mecanización. Precisamente el hecho de que una mayor mecanización reduzca la tasa de ganancia recoge la esencia de la «tendencia decreciente de la tasa de ganancia» en donde una mayor mecanización sería análoga al crecimiento de la «composición orgánica del capital» (Marx, 1894, pp. 269-341).

\section{EL EJÉRCITO LABORAL DE RESERVA Y SUS FUNCIONES}

Con la acumulación de capital puede crecer tanto el componente constante como variable del capital (Marx, 1867, p. 759). En el caso de crecer el capital variable (v'>0), surgen dos efectos, uno que influye sobre la clase asalariada y otro sobre los capitalistas.

Por un lado, asumiendo que las horas de trabajo son constantes,$^{18}$ el aumento del capital 
variable implica un aumento del salario y/o de los empleados. Incluso la acumulación podría empujar a que «la demanda de obreros supere su oferta, a raíz de lo cual los salarios pueden aumentar» (Marx, 1867, p. 760). Esto beneficia a la clase asalariada pues se da una «merma cuantitativa del trabajo impago que debe ejecutar el obrero» (Marx, 1867, p. 768). Por otro lado, si el salario real crece más que la "productividad laboral», ceteris paribus, las ganancias tenderán a disminuir (ver 4'). ${ }^{19}$ Es decir, si la acumulación lleva a un incremento «excesivo» del capital variable, las ganancias se «embotan», perjudicando a la clase capitalista (Marx, 1867, pp. 768-769).

Ambos efectos reflejan los intereses contradictorios entre asalariados y capitalistas - $y$, por ende, reflejan la lucha de clases en el capitalismo- - Sobre todo, para evitar la tendencia a la caída de las ganancias, el capitalismo necesita alguna «palanca» que disminuya los salarios y/o aumente la «productividad laboral» por encima del crecimiento salarial — sea con mayor mecanización y/o intensidad del trabajo- . Tales reacciones perjudican a los asalariados pues, por un lado, salarios más bajos restan capacidad de subsistencia y, por otro, una mayor «productividad laboral» alcanzada con mayor intensidad o mecanización podrían provocar que parte de los asalariados dejen necesitarse en la producción. Todas estas situaciones permiten entender que:

La acumulación capitalista produce de manera constante, antes bien, y precisamente en proporción a su energía y a su volumen, una población obrera relativamente excedentaria, esto es, excesiva para las necesidades medias de valorización del capital y por tanto superflua. (Marx, 1867, p. 784)

Así, el avance de la acumulación necesita de una sobrepoblación relativa: un grupo de no empleados o subempleados, sin ingresos o con ingresos bajos que no alcanzan la subsistencia promedio social. Estas personas son «excedentarias» para la acumulación (reemplazables por una mayor mecanización o mayor intensidad del trabajo de quienes se encuentran empleados) y hasta sufren de una explotación mayor a la media (reflejada en una contracción salarial o una mayor intensidad del trabajo). Junto a esta sobrepoblación relativa, emerge una población adecuada: grupo de empleados - o no empleados - que alcanzan una subsistencia igual o mejor al promedio social (Marx, 1857-1858, pp. 111-114; 1867, p. 784; Ricardo, 1821, pp. 303-7; Noroña y Cajas-Guijarro, 2014, p. 63).

Cuando la población adecuada está empleada dentro del capitalismo se transforma en un ejército laboral activo (ELA). En cambio, la sobrepoblación relativa funcional para la acumulación conforma un ejército laboral de reserva (ELR) (ver Marx, 1867, p. 798; cfr. Devine, 2005; Nun, 1969). El ELR agrupa a desempleados o subempleados que tienen la posibilidad de en algún momento - pasar al ejército laboral activo (Marx, 1867, p. 797; cfr. Trotsky, 1939, pp. 23-24) y cumple las funciones de brindar las personas necesarias para ajustar la producción según lo requiera la acumulación — función de reserva-, y aumentar la explotación sobre la clase trabajadora empujando los salarios a la baja o a una mayor intensidad del trabajo - funciones de explotación- (Quijano, 1973; Nun, 1969; Noroña y Cajas-Guijarro, 2014, pp. 63-64). Así, el ELR brinda «el material humano explotable y siempre disponible» para la acumulación (Marx, 1867, pp.786-7).

FUNCIÓN DE RESERVA. La función de reserva del ELR consiste en que éste brinda las personas 
necesarias para ajustar el empleo según las necesidades de la acumulación capitalista. En otras palabras:

Es necesario que se pueda volcar súbitamente grandes masas humanas en los puntos decisivos, sin que con ello se rebaje la escala alcanzada por la producción en otras esferas. La sobrepoblación proporciona [ejército laboral de reserva en el capitalismo, NDA] esas masas. (Marx, $1867, \mathrm{p} .787$ )

Igualmente, la función de reserva permite que el ELR absorba a todos quienes han perdido su empleo al ser reemplazados por los medios de producción gracias a la mecanización empujada por la propia acumulación. Cabe recordar que:

A medida que progresa la acumulación, pues, no solamente se da un acrecentamiento cuantitativo y simultánea de los diversos elementos reales del capital: [...] [también hay] cambios graduales en la composición técnica del capital [análoga a la mecanización, NDA], cuyo factor objetivo aumenta progresivamente [medios de producción, NDA], en magnitud relativa, frente al factor subjetivo [fuerza de trabajo, NDA]. (Marx, 1867, p. 773) ${ }^{20}$

El incremento continuo de los medios de producción respecto a la fuerza de trabajo puede representarse con un aumento permanente de la mecanización a una tasa constante $\gamma \mathrm{m}$-causada, entre otras cosas, por la propia competencia capitalista que empuja a la tecnificación «permanente» de la producción-. Esta idea se recoge en (11).

$$
\gamma_{m}=\frac{m^{\prime}}{m}
$$

Para deducir una versión analítica de la función de reserva que represente la adaptación del ELR a la dinámica de la acumulación, así como la absorción de las personas expulsadas por el crecimiento continuo de la mecanización, se asumirá que el ejército solo incluye desempleados - no existe subempleo- ${ }^{21}$ ). Esto se hace indirectamente definiendo una tasa de empleo (x) como la ratio entre empleados ( $\mathrm{E}$ ) y el total de población disponible para trabajar $(\mathrm{N})$, tal como plantea (12).

$$
x=\frac{E}{N}
$$

Aquí se asumirá que el crecimiento de la población disponible para trabajar es exógeno - pudiendo depender, por ejemplo, de aspectos demográficos—, el cual se representa con una tasa constante $\beta$, como indica (13).

$$
\frac{N^{\prime}}{N}=\beta, \quad \beta \geq 0
$$

Juntando (12) y (13) con (1), (2), (3) y (8) surge la expresión (RES) que representa a la función de reserva pues muestra los determinantes del aumento del empleo. ${ }^{22}$ 


$$
\frac{x^{\prime}}{x}=\left(\gamma_{K}-\beta\right)-\frac{m^{\prime}+m\left(p^{\prime} / p\right)+h\left(w^{\prime} / p\right)}{m+h(w / p)}
$$

Observando la función de reserva (RES) se nota que la tasa de empleo crecerá cuando crezca el capital total —es decir, cuando $\gamma \mathrm{K}>\mathrm{O}$-, mientras que se contraerá cuando la población disponible para trabajar aumente - es decir, cuando $\beta>0-$.

En el primer caso se puede pensar que la tasa de empleo crece gracias a que una mayor acumulación amplía la demanda de fuerza de trabajo; el segundo caso, en cambio, se puede interpretar como la tendencia a mayor desempleo por mayor población. Sobre el efecto de la mecanización, las horas de trabajo, los precios y el salario en la tasa de empleo, la situación es más compleja y se la deja para un análisis futuro. De todas formas, se ve que un incremento de la mecanización $-m^{\prime}>0-$ presiona a la reducción de la tasa de empleo.

FUNCIONES DE EXPLOTACIÓN (SALARIOS E INTENSIDAD). A más de ajustar la reserva, el ELR contribuye a ajustar la explotación a la clase asalariada. Esto sucede en especial porque sus miembros ofrecen su propia fuerza de trabajo por ingresos menores a los necesarios para la subsistencia social promedio - generando una contracción salarial—, además que presionan a que los empleados incrementen su intensidad del trabajo en respuesta, por ejemplo, a amenazas de despido y reemplazo (Marx, 1867, p. 791; Noroña y Cajas-Guijarro, 2014, p. 70). Así, hasta se puede hablar de dos funciones de explotación del ELR: una que reduce los salarios y otra que aumenta la intensidad del trabajo.

Como resultado de ambas funciones de explotación, cuando el ELR crece en términos relativos - es decir, con respecto al total de la población disponible para trabajar-, la explotación aumenta (Marx, 1867, p. 793), permitiendo a la clase capitalista:

Arrancar una cantidad determinada de trabajo de un número menor de obreros, en vez de extraerla, con la misma baratura e incluso a un precio más conveniente, de un número mayor» [haciendo que un mismo capital NDA] «ponga en movimiento más trabajo gracias a una explotación mayor en extensión e intensidad de las fuerzas de trabajo individuales. (Marx, 1867, p. 791)

Aunque la acumulación presione al aumento salarial, tal situación puede compensarse con incrementos en la intensidad del trabajo, pues la misma acumulación puede acrecentar «la oferta de obreros mediante su «puesta en libertad», mientras que a la vez la presión de los desocupados obliga a los ocupados a poner en movimiento más trabajo» (Marx, 1867, pp. 796-197). Así, las funciones de explotación permiten que las ganancias y la acumulación crezcan sin que necesariamente crezca el empleo ni los salarios en igual proporción (ver Marx, 1867, pp.629-643; pp. 776-7; Sweezy, 1942, p. 155).

Para representar estas ideas se proponen dos expresiones que recojan la presión del ELR a aumentar la intensidad del trabajo y a reducir los salarios. En el caso de la función de explotación vía intensidad, (14) indica que los trabajadores incrementarán su intensidad del trabajo precisamente cuando aumenta el peso relativo del ELR — equivalente a que reduzcan su intensidad cuando haya más empleo y, por tanto, el ELR es menor-. 


$$
e=f_{2}\left(x, w, \alpha_{20}\right), f_{2 x}<0, f_{2 w}>0
$$

La intención de (14) es que, cuando disminuye la tasa de empleo, la intensidad será mayor por la presión que los desempleados en reserva provocan sobre los empleados activos. Además, (14) recoge la posibilidad de que la intensidad también aumente al existir salarios más altos; es decir, con salarios más altos, los trabajadores podrían motivarse a trabajar con mayor intensidad (ver Shapiro y Stiglitz, 1984). ${ }^{23}$ A estos aspectos se agrega otros elementos ( $\alpha_{20}$ ) que también influyen en la intensidad, los cuales se asumen constantes.

En cambio, la función de explotación vía salarios se representa - con fines ilustrativos - usando una curva de Phillips de salario nominal - similar a la planteada por Goodwin (1967) o por las múltiples interpretaciones de Flaschel (2009) -, como se presenta en (15).

$$
\frac{w^{\prime}}{w}=f_{3}\left(x, \alpha_{30}\right), f_{3 x}>0
$$

El objetivo de (15) es representar que, a menor tasa de empleo - implicando mayor peso relativo del ELR - los salarios tenderán a decrecer, sea porque los desempleados ofrecen su fuerza de trabajo a salarios muy bajos o porque un mayor desempleo implica una menor capacidad organizativa de los trabajadores, debilitándose su poder de negociación (Phillips, 1958). Tal situación podría afectarse por otros factores (a30) que se asumen constantes.

Sobre al incremento salarial recogido en (15) cabe una reflexión adicional. Si los salarios nominales aumentan por un menor peso relativo del ELR, dicho movimiento podría tener un efecto relevante sobre los precios. Por ejemplo, ante un alza salarial los capitalistas podrían sentirse motivados a incrementar los precios de sus productos para que las ganancias - nominales - no disminuyan - aspecto que influye, a su vez, sobre el salario real-. Esta intuición se recoge en (16), de forma análoga a la propuesta de Desai (1978, p. 532) quien amplía el modelo de Goodwin incluyendo precios.

$$
\frac{p^{\prime}}{p}=f_{4}\left(p, w, q \cdot \alpha_{40}\right), f_{4 p}<0, f_{4 w}>0, f_{4 q}<0
$$

El objetivo de (16) es indicar que, a mayor salario nominal, existirá la tendencia a una mayor inflación - una mayor tasa de crecimiento de los precios-. A este efecto se agrega que, si los precios actuales ya son altos, entonces es probable que los precios futuros tiendan a caer — tendencia a la estabilidad de precios-. También se agrega que, si la "productividad laboral» es alta, ésta podría contribuir a una reducción de costos y, por tanto, de precios. Como siempre, se agregan otros aspectos (a40) que se asumen constantes. ${ }^{24}$

En resumen, (14) y (15) pueden considerarse funciones de explotación por intensidad y salarios respectivamente, mientras que (16) puede verse como una consecuencia causada por la función de explotación salarial sobre los precios e, indirectamente, sobre el salario real. 
INTERPRETANDO UNA DINÁMICA COMPLEJA

Al combinar los determinantes de la acumulación y de la tasa de ganancia con las funciones del ELR, su influencia sobre precios, así como el crecimiento permanente de la mecanización, es posible construir un sistema dinámico que describa el vínculo entre las variables —endógenas - tasa de empleo $(\mathrm{x})$, tasa de ganancia $(\mathrm{g})$, salarios $(\mathrm{w})$, precios $(\mathrm{p})$ y mecanización $(\mathrm{m})$ en el capitalismo (vínculo afectado por varios parámetros exógenos). Tal sistema, en su versión completa, se recoge en (A), (B), (C), (D) y (E). ${ }^{25}$

$$
\begin{gathered}
\frac{\boldsymbol{x}^{\prime}}{\boldsymbol{x}}=s \boldsymbol{g}-\left(\frac{\boldsymbol{m}^{\prime}+\boldsymbol{m}\left(\boldsymbol{p}^{\prime} / \boldsymbol{p}+\delta\right)+h\left(\boldsymbol{w}^{\prime} / \boldsymbol{p}\right)}{\boldsymbol{m}+h(\boldsymbol{w} / \boldsymbol{p})}+\beta\right) \\
\boldsymbol{g}=\frac{h\left\{f_{1}\left[f_{2}\left(\boldsymbol{x}, \boldsymbol{w}, \alpha_{20}\right), \boldsymbol{m}, \alpha_{10}\right]-\boldsymbol{w} / \boldsymbol{p}\right\}-\boldsymbol{m} \delta}{\boldsymbol{m}+h(\boldsymbol{w} / \boldsymbol{p})} \\
\frac{\boldsymbol{w}^{\prime}}{\boldsymbol{w}}=f_{3}\left(\boldsymbol{x}, \alpha_{30}\right) \quad(C) \\
\frac{\boldsymbol{p}^{\prime}}{\boldsymbol{p}}=f_{4}\left(\boldsymbol{p}, \boldsymbol{w}, f_{1}\left[f_{2}\left(\boldsymbol{w}, \boldsymbol{x}, \alpha_{20}\right), \boldsymbol{m}, \alpha_{10}\right] . \alpha_{40}\right)(D) \\
\frac{\boldsymbol{m}^{\prime}}{\boldsymbol{m}}=\gamma_{m} \quad(E)
\end{gathered}
$$

El sistema (A-E) es demasiado general pues aplica para cualquier tipo de funciones $\mathrm{f}_{1}, \mathrm{f}_{2}, \mathrm{f}_{3} \mathrm{y}$ $\mathrm{f}_{4}$ que cumplan (7), (14), (15) y (16) respectivamente. Tal sistema debe verse como molde teórico que permite experimentar con múltiples formas de dichas funciones. A su vez, (A-D) es un sistema complejo compuesto de múltiples elementos que interactúan de manera no lineal. Estas situaciones hacen necesario reducir el sistema a una versión menos compleja para entender la dinámica fundamental de las variables endógenas y obtener una interpretación relevante. Para cumplir dicho objetivo, se plantean algunos supuestos:

- Toda la ganancia se destina a la acumulación, es decir, $s=1$

- Las horas de trabajo se normalizan, es decir, $\mathrm{h}=1$

- No hay crecimiento de la población disponible para trabajar, es decir, $\beta=0$

- No hay depreciación del capital constante, es decir, $\delta=0$

- La mecanización crece a una tasa constante, pero baja, de $\gamma \mathrm{m}=10^{-4}$

- La «productividad laboral» depende de la mecanización y la intensidad del trabajo asumiendo una sustitución constante entre ambas, mientras que los demás factores no influyen. Una forma de formalizar esta idea es asumir una función de producción ${ }^{26}$ como la descrita en (8').

$$
q=f_{1}\left(e, m, \alpha_{10}\right)=e m
$$

- La intensidad depende positivamente de los salarios y negativamente del empleo mientras que otros factores hacen que ésta se mantenga en un nivel estándar. Eso se recoge en la función lineal (15’). 


$$
e=f_{2}\left(x, w, \alpha_{20}\right)=1+w-x
$$

- Los salarios nominales tienden a caer a una tasa autónoma de $25 \%$, a la vez y al mismo tiempo que tienden a crecer en $0,35 \%$ por cada punto porcentual registrado en la tasa de empleo, siguiendo una curva de Phillips análoga a la utilizada por Goodwin (1967). ${ }^{27}$ Tal curva se recoge en $\left(16^{\prime}\right)$.

$$
\frac{w^{\prime}}{w}=f_{3}\left(x, \alpha_{30}\right)=-0.25+0.35 x
$$

- La inflación cae al crecer los precios actuales y/o la «productividad», y crece al aumentar el salario nominal, siguiendo la propuesta de Desai (1978). Esto se recoge en (17’).

$$
\frac{p^{\prime}}{p}=f_{4}\left(p, w, q \cdot \alpha_{40}\right)=1-p+w-q
$$

Con estos supuestos se obtiene un sistema simplificado que captura la esencia de la presente interpretación de la «ley general de la acumulación capitalista», dejando de lado la complejidad que será analizada a futuro. Así, el modelo simplificado se recoge en (A'), (B'), (C'), (D') y (E'). ${ }^{28}$

$$
\begin{gathered}
\boldsymbol{g}=\frac{(1+\boldsymbol{w}-\boldsymbol{x}) \boldsymbol{m}-(\boldsymbol{w} / \boldsymbol{p})}{\boldsymbol{m}+(\boldsymbol{w} / \boldsymbol{p})} \quad\left(B^{\prime}\right) \\
\frac{\boldsymbol{w}^{\prime}}{\boldsymbol{w}}=-0.25+0.35 x \quad\left(C^{\prime}\right) \\
\frac{\boldsymbol{p}^{\prime}}{\boldsymbol{p}}=\mathbf{1}-\boldsymbol{p}+\boldsymbol{w}-(1+\boldsymbol{w}-\boldsymbol{x}) \boldsymbol{m} \quad\left(D^{\prime}\right) \\
\frac{\boldsymbol{m}^{\prime}}{\boldsymbol{m}}=10^{-4} \quad\left(E^{\prime}\right)
\end{gathered}
$$

Para interpretar la dinámica del sistema (A'-D') se usan simulaciones numéricas ${ }^{29}$ usando los valores iniciales descritos en (I).

$$
x_{0}=0.5, w_{0}=0.5, p_{0}=0.5, m_{0}=1
$$

Al aplicar las simulaciones es posible estimar las trayectorias, en el tiempo, de salario, tasa de ganancia, tasa de empleo, precios y mecanización (ver Figura 1A). Como resultado se ve que tales variables son cíclicas, excepto la mecanización - que crece de forma continua-. En particular, se nota que la tasa de ganancia fluctúa entre etapas de ganancias — animación - y pérdidas - crisis-. Otro resultado llamativo es que, en un inicio las variables muestran ciclos con 
Figura 1a: Trayectorias del sistema (A'-D') con $\gamma \mathrm{m}=10-4.1 \mathrm{~A}$ : En el tiempo

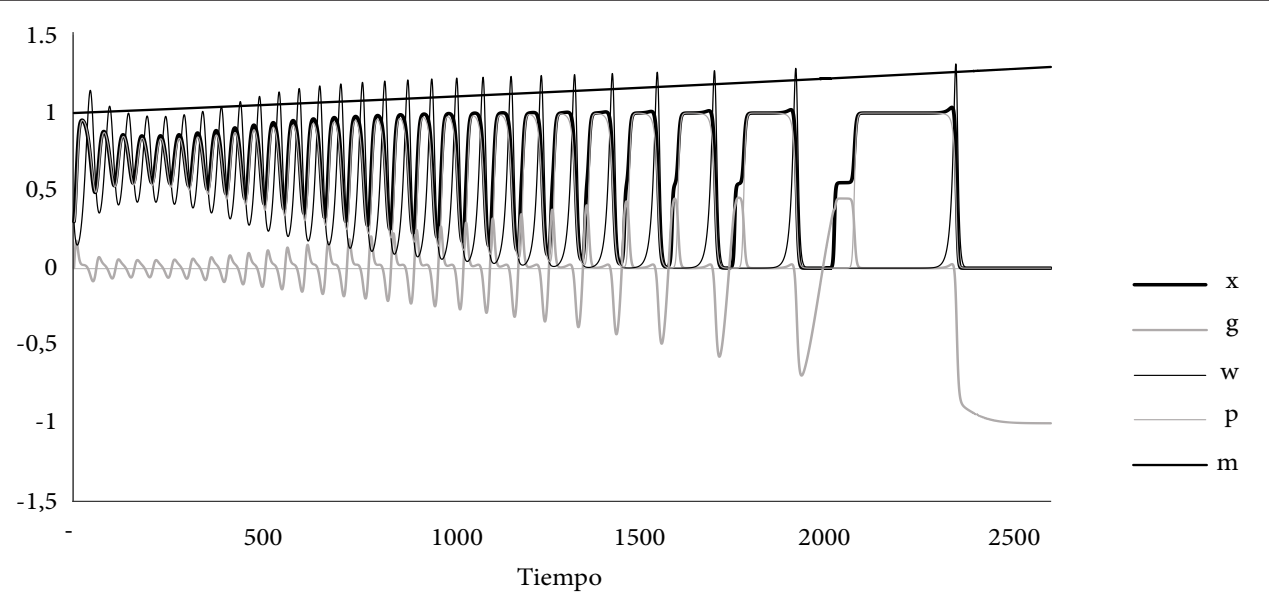

Nota: $x$ : tasa de empleo; g: tasa de ganancia; w: salarios; p: precios; m: mecanización. Fuente: estimación propia del sistema (A’-D’) con valores iniciales descritos en (I).

Figura 1b. Trayectorias del sistema (A'-D') con $\gamma \mathrm{m}=10-4$. 1B: En el espacio $\mathrm{x}-\mathrm{w}-\mathrm{m}$

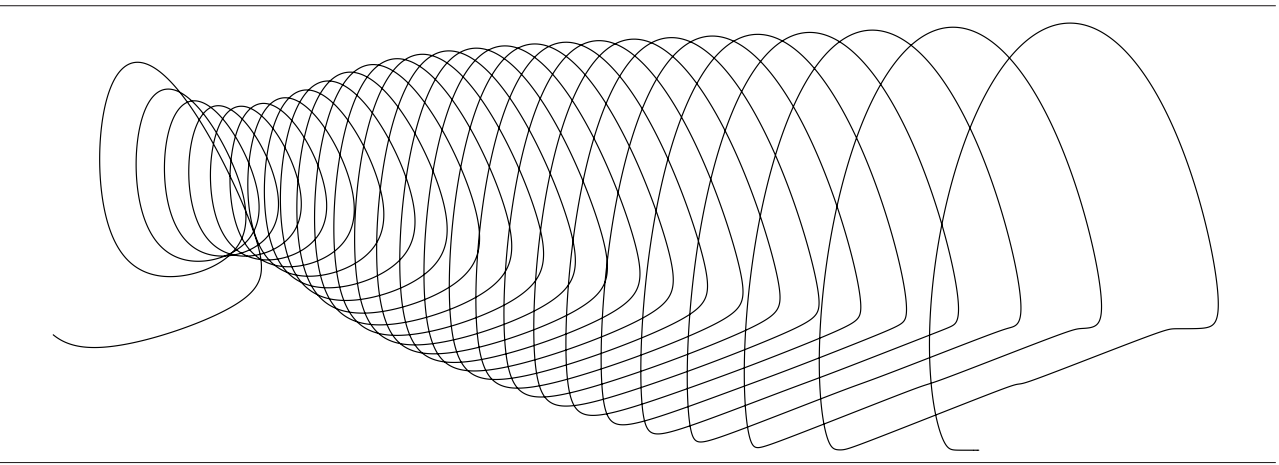

Nota: x: tasa de empleo; g: tasa de ganancia; w: salarios; p: precios; m: mecanización. Fuente: estimación propia del sistema (A’-D’) con valores iniciales descritos en (I).

amplitudes decrecientes, pero al pasar el tiempo - y aumentar la mecanización — la tendencia cambia y los ciclos pasan a tener amplitudes crecientes. Así, mientras a corto plazo los ciclos son «leves», a largo plazo son cada vez más «fuertes», destacándose incluso que en cada nuevo ciclo son más graves las pérdidas sobre la tasa de ganancia. De hecho, las pérdidas se vuelven tan agudas que el modelo colapsa - llegando a puntos con empleo, salario y precio prácticamente nulos-. Esta combinación de una temporada de ciclos de amplitud decreciente y otra de ciclos de amplitud creciente se refleja en la peculiar forma que las trayectorias forman en el espacio compuesto por empleo, salarios y mecanización (ver Figura 1B).

Tales resultados hacen pensar, por un lado, que el vínculo entre acumulación de capital y ELR - que lleva implícita la contradicción capital-salario- genera ciclos al estilo de Goodwin (1967). Sin embargo, por otro lado, el aumento permanente de la mecanización parece provocar 
que, en algún momento, se pase de una etapa de ciclos de amplitud decreciente a otra con amplitud creciente, en donde cada nuevo ciclo hace que la tasa de ganancia sufra pérdidas cada vez más fuertes hasta que el modelo colapsa. Esta interpretación del efecto de la mecanización sobre el sistema (A'-D') parece adecuada, pues si se hacen nuevas simulaciones asumiendo que la mecanización es constante $\gamma \mathrm{m}=\mathrm{o}$ y manteniendo inalterados los demás supuestos, el resultado es que los ciclos pierden amplitud -igualmente las pérdidas en la tasa de ganancia son cada vez menores - y el sistema termina en un punto atractor (ver Figura 2)..$^{\circ}$

\section{DE LA LEY GENERAL DE LA ACUMULACIÓN CAPITALISTA}

A UNA TENDENCIA CÍCLICA-DECRECIENTE DE LA TASA DE GANANCIA

Los resultados - preliminares - obtenidos de las simulaciones del sistema (A'-E') hacen pensar que la dinámica cíclica es propia del capitalismo, lo cual no contradice - y hasta podría reforzar- la tendencia a que, en el largo plazo, una mayor mecanización genere caídas cada vez más graves en la tasa de ganancia. Tal comportamiento parece venir de la interacción entre los determinantes de la tasa de ganancia y la acumulación junto con las funciones del ELR. Dicha dinámica evidenciaría una de las contradicciones fundamentales del capitalismo: la búsqueda de ganancias explotando a la fuerza de trabajo, lo cual lleva a que sean las luchas entre trabajadores y capitalistas las que generen ciclos (ver Goodwin, 1967) - y violencia - exacerbados a medida que el capitalismo amplía sus fuerzas productivas. Además, ese comportamiento cíclico parece variar entre el corto y el largo plazo, aspecto que podría vincularse a los llamados ciclos económicos largos, es decir, ciclos de grandes proporciones que duran hasta décadas (Marx, 1867, p. 787; Kondratiev, 1935, p. 105; Mandel, 1979, pp. 65-66; Solow, 1990, pp. 8-9; Noroña y Cajas-Guijarro, 2014, p. 74).

Estos ciclos de la acumulación estarían compuestos por etapas de animación y de crisis (Marx, 1867, p. 787; Noroña y Cajas-Guijarro, 2014, p. 73) bajo la siguiente lógica:

Etapa de animación: asumiendo una situación inicial de aumento de la explotación laboral, ésta permite que se amplíen las ganancias capitalistas, se amplíe la tasa de ganancia y, por tanto, se acelere la acumulación pues cada vez hay más dinero disponible para que la clase capitalista adquiera medios de producción y fuerza de trabajo. Esa ampliación de la fuerza de trabajo empleada absorbe a parte de los miembros del ELR, reduciéndose su peso relativo y permitiendo que la clase trabajadora se fortalezca, pudiendo así exigir salarios más altos creando mayores presiones inflacionarias según la reacción capitalista - junto con exigir una menor intensidad del trabajo en la producción - funciones de explotación en favor de la clase trabajadora-, todo lo cual reduce la explotación laboral a futuro.

Etapa de crisis: asumiendo una situación inicial de reducción de la explotación laboral, ésta hace que las ganancias se estanquen y hasta se contraigan, contrayéndose también la tasa de ganancia; al caer las ganancias, la acumulación se merma pues los capitalistas cuentan con menos dinero para adquirir medios de producción y fuerza de trabajo, implicando un menor empleo. Al caer el empleo se amplía el ELR pues se expulsan trabajadores que no logran emplearse por la acumulación a la baja - función de reserva hace que las personas sean expulsadas por la merma de la acumulación-; el mayor peso relativo del ELR debilita a la clase trabajadora, brindando a los capitalistas el poder de reducir los salarios — reduciendo las presiones inflacionarias según 
Figura 2. Trayectorias en el tiempo del sistema (A'-D') con $\gamma \_m=0$

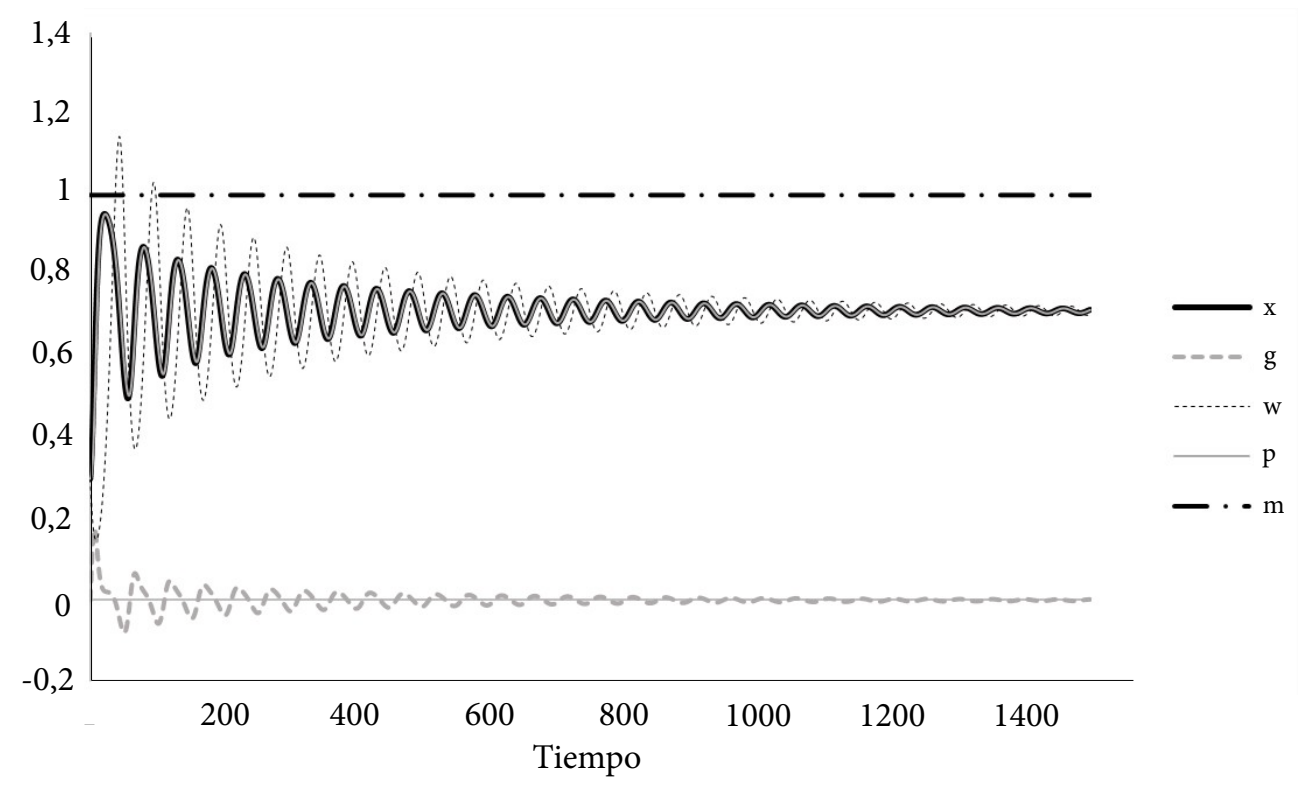

Fuente: estimación propia del sistema (A'-D’) con valores iniciales descritos en (I) y con mecanización constante.

Figura 3. Posible vínculo acumulación de capital-ejército laboral de reserva

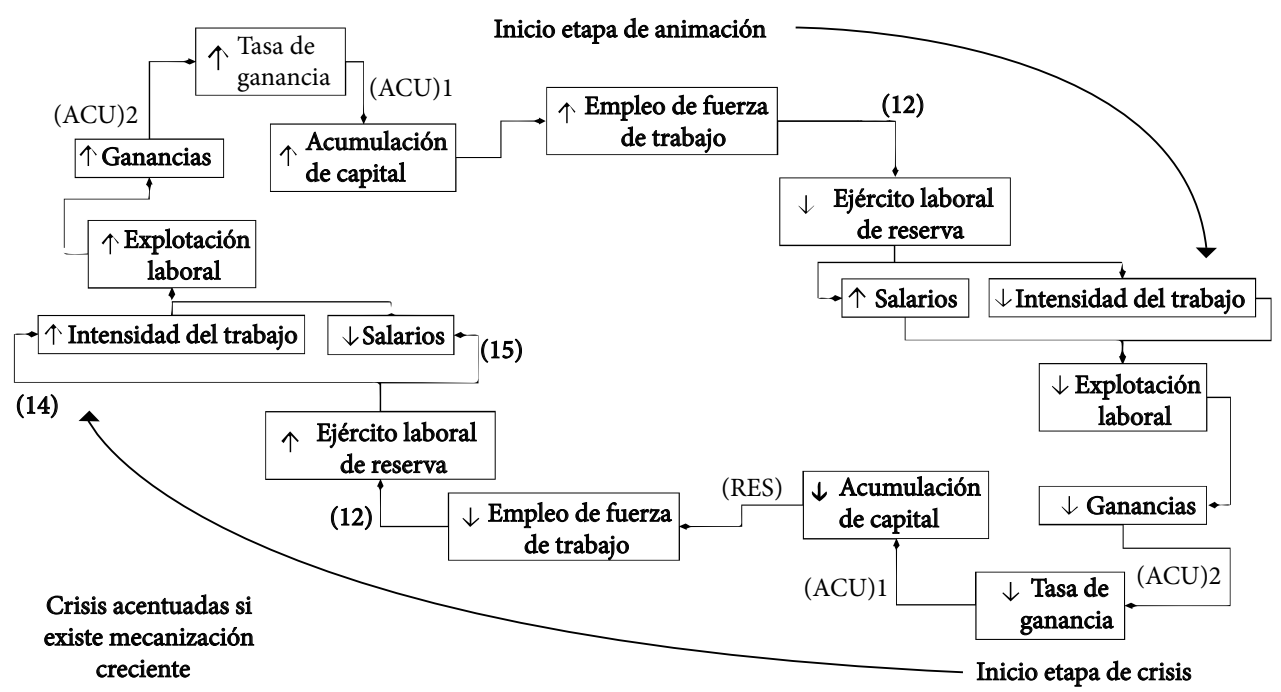

Nota: Los números en paréntesis indican las expresiones que conectan a cada elemento. Fuente: elaboración propia a partir de Marx (1867, cap. XXIII). 
cómo ellos reaccionen - y exigir una mayor intensidad de trabajo al poner en competencia a empleados y desempleados, todo lo cual aumenta la explotación laboral a futuro.

Dicha dinámica cíclica afecta tanto al empleo, los salarios y especialmente a la tasa de ganancia. Pero el comportamiento cíclico es peculiar pues, al crecer la mecanización, la amplitud de los ciclos decrece a corto plazo y se amplía a largo plazo, provocando en este último caídas cada vez más drásticas de la tasa de ganancia, pero durante las etapas de crisis. A este comportamiento se lo podría llamar como una «tendencia cíclica-decreciente de la tasa de ganancia». Así, las etapas de animación y crisis están conectadas, formando un solo gran proceso cíclico que surgiría del vínculo entre acumulación y ELR, además de ser exacerbado por el efecto de la mecanización sobre la tasa de ganancia (ver Figura 3).

En medio de este complejo comportamiento, se da una continua atracción y repulsión de la sobrepoblación relativa según cómo se amplía o contrae la «riqueza» generada por la acumulación. Este resultado permite el surgimiento de la «ley general de la acumulación capitalista», que señala lo siguiente:

Cuanto mayores sean la riqueza social, el capital en funciones, el volumen y vigor de su crecimiento y, por tanto, también, la magnitud absoluta de la población obrera y la fuerza productiva de su trabajo, tanto mayor será la pluspoblación relativa o ejército industrial de reserva. La fuerza de trabajo disponible se desarrolla por las mismas causas que la fuerza expansiva del capital. La magnitud proporcional del ejército industrial de reserva, pues, se acrecienta a la par de las potencias de la riqueza. Pero cuanto mayor sea este ejército de reserva en proporción al ejército obrero activo, tanto mayor será la masa de la pluspoblación consolidada o las capas Cuanto mayores sean la riqueza social, el capital en funciones, el volumen y vigor de su crecimiento y, por tanto, también, la magnitud absoluta de la población obrera y la fuerza productiva de su trabajo, tanto mayor será la pluspoblación relativa o ejército industrial de reserva. La fuerza de trabajo disponible se desarrolla por las mismas causas que la fuerza expansiva del capital. La magnitud proporcional del ejército industrial de reserva, pues, se acrecienta a la par de las potencias de la riqueza. Pero cuanto mayor sea este ejército de reserva en proporción al ejército obrero activo, tanto mayor será la masa de la pluspoblación consolidada o las capas obreras cuya miseria está en razón inversa a la tortura de su trabajo. Cuanto mayores sean, finalmente, las capas de la clase obrera formadas por menesterosos enfermizos y el ejército industrial de reserva, tanto mayor será el pauperismo oficial. Ésta es la ley general, absoluta, de la acumulación capitalista. (Marx, 1867, p. 803)

Interpretada de otra forma, esta «ley» ${ }^{31}$ podría implicar que la existencia del ELR es vital para la continuidad de la acumulación, pues sus funciones de reserva y de explotación evitan que la acumulación colapse ante una reducción permanente de la explotación laboral que podría darse si no fuera factible expulsar trabajadores de la producción y que dichos trabajadores expulsados contribuyan a reducir salarios y/o a aumentar la intensidad. Sin embargo, a medida que la mecanización crece cada vez más, las funciones del ELR al parecer actúan como una fuerza que cíclicamente "contrarresta» la caída de la tasa de ganancia (similar a lo que sugeriría Marx, 1894, pp. 302-303). Pero, a largo plazo, las funciones antes que evitar, posiblemente se complementan con la tendencia decreciente de la tasa de ganancia ante el incremento de la tecnificación, dando como resultado que las pérdidas existentes en las crisis sean cada vez más graves. 
Por tanto, de la sucesión de etapas de animación y crisis — mediada por la mecanización — se puede concluir que «el propio mecanismo del proceso capitalista de producción remueve los obstáculos que genera transitoriamente» (Marx, 1867, p. 769). En particular, sería gracias al aumento de la explotación laboral que el capitalismo logra salir de sus crisis. Sin embargo, el crecimiento de la mecanización parece provocar que, a largo plazo, los ciclos adquieran una amplitud creciente, lo cual en el caso de la tasa de ganancia implica que, en cada nueva crisis, las pérdidas sean cada vez mayores. Semejante resultado muestra que el proceso cíclico del capitalismo es en extremo volátil pues, según cómo se dé el choque de intereses entre trabajadores y capitalistas en crisis, podría surgir una situación extrema en donde las protestas trabajadoras que emerjan ante al aumento de la explotación laboral — para rescatar a la tasa de ganancia de su caída - lleven a que el capitalismo colapse — de forma más compleja que el colapso del modelo de este artículo-. En cierta forma se justificaría la intuición de que el capitalismo —aun siendo cíclico- lleva dentro de sí las «semillas de su propia destrucción» (Marx, 1850).

Finalmente, cabe indicar que los resultados son apenas ilustrativos y el real vínculo entre ELR, acumulación y tasa de ganancia es más complejo según cada sociedad capitalista histórica-concreta estudiada —-más aún en el capitalismo periférico- . Es por ello que el modelo aún necesita, a futuro, contrastarse con datos empíricos y supuestos más realistas. En todo caso, la formalización de la condición cíclica del capitalismo parece una reinterpretación útil de la ley general de la acumulación y podría complementarse con muchas otras interpretaciones disponibles (Goodwin, 1967, pp. 54-58; Shaikh, 1990, pp. 233-4; Devine, 2005, p. 15; Flaschel, 2009, pp. 107-47; Basu et al., 2012, pp. 1-3; Noroña y Cajas-Guijarro, 2014, p. 75).

\section{CONCLUSIÓN}

En este artículo se ha presentado una reinterpretación analítica de la llamada «ley general de la acumulación capitalista»; una «ley» que, según la propuesta de este trabajo, permitiría entender que la dinámica cíclica del capitalismo surge del vínculo entre la acumulación de capital y el ejército laboral de reserva. Asimismo, una «ley» que parece sugerir que la tendencia a la caída de la tasa de ganancia causada por una mayor mecanización también podría ser cíclica. Para abordar dicha reinterpretación se ha construido un sistema dinámico cuya simulación numérica - aún preliminar - ha brindado evidencia a favor de una interpretación «cíclica» de la «ley» postulada por Marx, así como evidencia de que el crecimiento de la mecanización tiende a agudizar las etapas de crisis en el largo plazo.

Si bien la reinterpretación se ha hecho en términos analíticos, aún sus resultados no pueden aceptarse como generales e inherentes al capitalismo. A más de profundizar sobre las propiedades del modelo, hay varias extensiones importantes que deberían hacerse: uso de esquemas de reproducción ampliada en donde cada departamento tenga su propio nivel de precios y pueda incurrir en desequilibrios; capacidad de ahorro de trabajadores; diferentes tipos de ELR, sobre todo subempleados y desempleados con diferentes funciones de reserva y de explotación; el papel de los procesos de concentración y centralización del capital; el papel de una interpretación analítica de la teoría del valor — sea desde alguna de las múltiples interpreta-

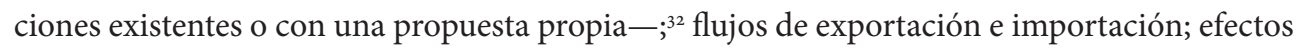


monetarios sobre precios y salarios, incluyendo el papel de las expectativas; el papel de las finanzas y la especulación; etc.

Pero quizá aún más importante que las posibles extensiones teóricas, sería crucial que también se busquen mecanismos para estimar empíricamente la forma específica del modelo y sus parámetros para sociedades capitalistas histórico-concretas. El fin de dicho ejercicio sería verificar hasta qué punto el modelo teórico y sus predicciones se corresponden con los ciclos capitalistas vividos a lo largo de la historia, a fin de mejorar nuestra comprensión del capitalismo, una «civilización de la desigualdad» — como diría Schumpeter- que encierra en su seno profundas y complejas contradicciones que deben abordarse desde el máximo número de visiones posibles. Contradicciones que incluso pueden causar que, con el tiempo, las etapas de crisis sean cada vez más extremas.

Los resultados de este artículo ojalá aporten a mejorar nuestra comprensión sobre el capitalismo, pero también hay una pretensión política clara: comprender cómo actúa el capitalismo debería servir para entender cómo superarlo. Posiblemente una idea a destacar es que la acumulación de capital solo puede existir en tanto que el ELR presiona a las personas empleadas. Es decir, la existencia de la acumulación depende de la capacidad del capitalismo de dividir a la clase trabajadora. La lección política de este resultado teórico es que la clase trabajadora debe buscar mecanismos para mantener unidos no solo a quienes logran entrar en el sistema como empleados, sino también a quienes son excluidos de éste y caen en el ELR. Dicha unión es aún más urgente si, a largo plazo, las nuevas crisis que surjan generen pérdidas más extremas que exigirán, a su vez, una explotación más extrema a la fuerza de trabajo —e incluso a la naturaleza - para que el capitalismo continúe existiendo.

Todavía queda mucho camino por recorrer antes de que la clase trabajadora logre unir en una misma fuerza a empleados y excluidos en su lucha frente a la explotación capitalista más aún en el capitalismo periférico-, al menos la obra de Marx dejó algunas pistas para entender las consecuencias de la desunión: la existencia de crisis recurrentes - y cada vez más extremas- que son superadas exacerbando la explotación.

\section{NOTAS}

1 Se agradece a William Sacher, Giovanny Manosalvas y a dos evaluadores anónimos quienes, con sus sugerencias y comentarios a versiones preliminares de este artículo, ayudaron a mejorarlo notablemente. 2 Aspectos que podrían reinterpretarse, quizá siguiendo a Morishima (1973).

3 Versiones preliminares - e inconclusas- se encuentran en Noroña y Cajas-Guijarro (2014) y en CajasGuijarro (2012).

4 En alusión al término, en inglés, de «reserve army of labour».

5 Se asume que toda la capacidad instalada es utilizada.

6 Se asume una sola mercancía utilizable como medio de producción y de consumo. Una ampliación al modelo podría hacerse, por ejemplo, con los esquemas de reproducción ampliada (Marx, 1885), en donde cada departamento tenga su propio nivel de precios.

7 Se asume que en el capital constante solo existe un elemento fijo y no uno circulante (Marx, 1867, p. 252, 1885 , p. 484; Noroña y Cajas-Guijarro, 2014, p. 27).

8 La intensidad del trabajo es el desgaste promedio de fuerza de trabajo hecho en cada unidad de tiempo trabajado (Marx, 1867, p. 498; Mavroudeas et al., 2011, pp. 430-435) similar al «esfuerzo» de los modelos 
de salarios de eficiencia (Shapiro y Stiglitz, 1984).

9 Donde fij $=\partial \mathrm{fi} / \partial \mathrm{j}$.

10 «El dinero, en cuanto posee la propiedad de comprarlo todo, en cuanto posee la propiedad de apropiarse de todos los objetos es, pues, el objeto por excelencia. La universalidad de su cualidad es la omnipotencia de su esencia; vale, pues, como ser omnipotente» (Marx, 1844).

11 Deducción de (4').

Dada la definición de ganancias de $(4) \mathrm{G}=\mathrm{pQ}-(\mathrm{v}+\delta \mathrm{c})$, en ésta se puede insertar la definición de $\mathrm{Q}$ planteada en (6) ( $\mathrm{Q}=\mathrm{qhE})$, la definición de v planteada en $(3)(\mathrm{v}=$ whE) y la definición de c planteada en $(2)(C=P a)$. Como resultado se obtiene $(4 a)$.

$$
G=p q h E-(w h E+\delta p A)(4 a)
$$

Si se divide ambos lados de (4a) para el producto phe y se inserta la definición de mecanización recogida en $(8)(\mathrm{m}=\mathrm{A} / \mathrm{E})$ se obtiene $(4 \mathrm{~b})$.

$$
\frac{G}{p h E}=q-\left(\frac{w}{p}+\frac{\delta m}{h}\right)
$$

Como h, E y p son positivos, entonces g y g serán positivas si y solo si se cumple (4'):

$$
q>\frac{w}{p}+\delta\left(\frac{m}{h}\right)
$$

12 Intuición cercana a la «voluntad de poder» (Nietzsche, 1891).

13 Asumiendo una sociedad capitalista cerrada, es decir, sin vínculos económicos con otras sociedades (Marx, 1867, p. 715, nota al pie). Para el caso de sociedades capitalistas abiertas, el modelo podría extenderse siguiendo la propuesta de Kalecki (1954).

14 Una extensión del modelo podría hacerse asumiendo que no todo el ahorro de las ganancias financia la acumulación debido a que los desequilibrios entre departamentos que producen medios de producción y de consumo (Marx, 1885, pp. 483-484; p. 548) a las decisiones de «inversión» -e incluso a los precios-.

15 Se asume que los asalariados no ahorran.

16 Como sugiere Goodwin (1990) tal representación es aceptable pues en una sociedad se dan tantas actividades económicas de forma casi simultánea, haciendo que la distinción discreto-continuo pueda asumirse irrelevante a nivel macro.

17 Deducción de (ACU1) y (ACU2).

Tomando la definición de tasa de crecimiento del capital, recogida en (10) $\gamma \mathrm{K}=\mathrm{K} / \mathrm{K}$, incluyendo el supuesto sobre la acumulación recogido en $(9)\left(\mathrm{K}^{\prime}=\mathrm{sG}-\delta \mathrm{C}\right)$, incluyendo la tasa de ganancia de $(5)(\mathrm{g}=$ $\mathrm{G} / \mathrm{K})$, reemplazando $\mathrm{K}$ por la definición planteada en $(1)(\mathrm{K}=\mathrm{C}+\mathrm{v})$, e insertando $(2)(\mathrm{c}=\mathrm{pA})$ y $(3)(\mathrm{v}=$ whE), se obtiene (ACU1').

$$
\gamma_{K}=s g-\frac{\delta p A}{p A+w h E}
$$

Si se divide numerador y denominador de la fracción que surge en (ACU1') para pE, y se incluye la mecanización planteada en $(8)(\mathrm{m}=\mathrm{A} / \mathrm{E})$, se obtiene (ACU1).

$$
\gamma_{K}=s g-\frac{\delta m}{m+h(w / p)}
$$

Aquí $\mathrm{g}=\mathrm{G} / \mathrm{k}$ puede descomponerse al reemplazar G por $(4 \mathrm{a})(\mathrm{G}=\mathrm{pqhE}-(\mathrm{whE}+\delta \mathrm{pA}))$ y usando las expresiones (1), (2) y (3), obteniéndose (ACU2').

$$
g=\frac{p q h E-(w h E+\delta p A)}{p A+w h E}
$$


Si se divide numerador y denominador del lado derecho de (ACU2') para pE, y se incluye la mecanización planteada en (8), se obtiene (ACU2).

$$
g=\frac{h(q-w / p)-m \delta}{m+h(w / p)}
$$

18 Por tanto, cualquier ampliación de la fuerza de trabajo desgastada por persona empleada se da con mayor intensidad del trabajo.

19 Tal movimiento puede pensarse como una reducción del plusvalor relativo, generado de la diferencia entre «productividad laboral» y salarios (Marx, 1867, p. 383).

20 Marx distinguió entre «composición técnica del capital» —vínculo material entre medios de producción y fuerza de trabajo- $\mathrm{y}$ «composición de valor del capital» — aproximación en valor de la primera(Marx, 1867, cap. XxiII, nota a). Como la mecanización no incluye precios, lo adecuado es tomarla como análoga de la «composición técnica del capital».

21 Se podría ampliar el modelo incluyendo la intuición de que subempleados y desempleados tendrían diferentes funciones de reserva y de explotación.

22 Deducción de (RES).

Derivando el logaritmo de (12) $(\mathrm{x}=\mathrm{E} / \mathrm{N})$, e insertando $(13)\left(\mathrm{N}^{\prime} / \mathrm{N}=\beta\right)$ se obtiene (RES1).

$$
\frac{x^{\prime}}{x}=\frac{E^{\prime}}{E}-\beta
$$

En E'/E hay influencia de la acumulación, así como de la mecanización, por lo que se incluye ambos factores en (RES1). Sobre la acumulación, se puede tomar $(1)(\mathrm{K}=\mathrm{C}+\mathrm{V})$, insertar $(2)(\mathrm{C}=\mathrm{pA})$ y $(3)(\mathrm{V}=$ whe), multiplicar y dividir para e y aplicar $(8)(\mathrm{m}=\mathrm{A} / \mathrm{E})$. Como resultado surge (RES2).

$$
K=E(m p+h w)(R E S 2)
$$

Aplicando logaritmos a (RES2), derivándola y recordando (10) $\left(\gamma \mathrm{K}=\mathrm{K}^{\prime} / \mathrm{K}\right)$, se obtiene (RES3) (asumiendo h constante).

$$
\gamma_{K}=\frac{E^{\prime}}{E}+\frac{p^{\prime} m+p m^{\prime}+w^{\prime} h}{m p+h w}
$$

Despejando E'/E de (RES3) e insertando en (RES1) y reacomodando términos, surge (RES).

$$
\frac{x^{\prime}}{x}=\left(\gamma_{K}-\beta\right)-\frac{m^{\prime}+m\left(p^{\prime} / p\right)+h\left(w^{\prime} / p\right)}{m+h(w / p)}
$$

23 Esto permite combinar, a futuro, el modelo expuesto con la idea de «salario de eficiencia».

24 Entre esos aspectos adicionales podría incluirse temas monetarios o los efectos de los desequilibrios de mercado sobre los precios - por ejemplo, una oferta mayor a la demanda en el mercado de mercancías puede llevar a una caída de precios y viceversa-.

25 Deducción de (A-E).

Tomando (RES) de la nota 22 e insertando (ACU1) de la nota 17 se obtiene (A). En cuanto a la tasa de ganancia, si se toma (ACU2) de la nota 17, se ve que dentro de ésta se encuentra, la cual está definida en ( 7$)\left(q=f_{1}\left(e, m, \alpha_{10}\right)\right)$. Si dentro de $(7)$ se inserta $(14)\left(e=f_{2}\left(x, w, \alpha_{2} O\right)\right)$, se tiene $q=f_{1}\left[f_{2}\left(x, w, \alpha_{2} O\right)\right.$, $\mathrm{m}, \alpha_{10}$ ]. Reemplazando este resultado en (ACU2) surge (B). Usando (А) y (в), junto con (15) $\left(\mathrm{w}^{\prime} / \mathrm{w}=\mathrm{f}_{3}\right.$ $\left.\left(\mathrm{x}, \alpha_{3} \mathrm{O}\right)\right),(16)\left(\mathrm{p}^{\prime} / \mathrm{p}=\mathrm{f}_{4}\left(\mathrm{p}, \mathrm{w}, \mathrm{q}, \mathrm{a}_{4} \mathrm{O}\right)\right)$ y $(11)\left(\mathrm{m}^{\prime} / \mathrm{m}=\gamma \mathrm{m}\right)$ surge el sistema $(\mathrm{A}-\mathrm{E})$ (donde se resaltan las variables endógenas).

$$
\frac{\boldsymbol{x}^{\prime}}{\boldsymbol{x}}=s \boldsymbol{g}-\left(\frac{\boldsymbol{m}^{\prime}+\boldsymbol{m}\left(\boldsymbol{p}^{\prime} / \boldsymbol{p}+\delta\right)+h\left(\boldsymbol{w}^{\prime} / \boldsymbol{p}\right)}{\boldsymbol{m}+h(\boldsymbol{w} / \boldsymbol{p})}+\beta\right)
$$




$$
\begin{gathered}
\boldsymbol{g}=\frac{h\left\{f_{1}\left[f_{2}\left(\boldsymbol{x}, \boldsymbol{w}, \alpha_{20}\right), \boldsymbol{m}, \alpha_{10}\right]-\boldsymbol{w} / \boldsymbol{p}\right\}-\boldsymbol{m} \delta}{\boldsymbol{m}+h(\boldsymbol{w} / \boldsymbol{p})} \\
\frac{\boldsymbol{w}^{\prime}}{\boldsymbol{w}}=f_{3}\left(\boldsymbol{x}, \alpha_{30}\right) \quad(C) \\
\frac{\boldsymbol{p}^{\prime}}{\boldsymbol{p}}=f_{4}\left(\boldsymbol{p}, \boldsymbol{w}, f_{1}\left[f_{2}\left(\boldsymbol{w}, \boldsymbol{x}, \alpha_{20}\right), \boldsymbol{m}, \alpha_{10}\right] \cdot \alpha_{40}\right) \quad(D) \\
\frac{\boldsymbol{m}^{\prime}}{\boldsymbol{m}}=\gamma_{m} \quad(E)
\end{gathered}
$$

26 La expresión (8’) es una versión con elasticidades unitarias de una función Cobb-Douglas. Se la usa pues es una manera fácil de representar sustitución constante entre intensidad y mecanización manteniendo la producción constante.

27 Los valores planteados en este supuesto solo se usaron con fines ilustrativos pues generan resultados visuales nítidos. De todas formas, dichos valores no son descabellados, aunque lo más adecuado sería verificarlos empíricamente.

28 Deducción de (A'-E').

Dado el sistema (A-E) de la nota 25, se busca simplificarlo usando los supuestos (s) y las expresiones ( $7^{\prime}$ ), $\left(14^{\prime}\right),\left(15^{\prime}\right)$ y $\left(16^{\prime}\right)$.

$$
\begin{gathered}
s=1, \quad h=1, \quad \beta=0, \quad \delta=0, \quad \gamma_{m}=0.0001=10^{-4} \\
q=f_{1}\left(e, m, \alpha_{10}\right)=e m \quad\left(7^{\prime}\right) \\
e=f_{2}\left(x, w, \alpha_{20}\right)=1+w-x \quad\left(14^{\prime}\right) \\
\frac{w^{\prime}}{w}=f_{3}\left(x, \alpha_{30}\right)=-0.25+0.35 x \quad\left(15^{\prime}\right) \\
\frac{p^{\prime}}{p}=f_{4}\left(p, w, q . \alpha_{40}\right)=1-p+w-q \quad\left(16^{\prime}\right)
\end{gathered}
$$

Un elemento útil para la simplificación es q, la cual puede simplificarse insertando en ( $\left.7^{\prime}\right)$ el resultado de (14'), generándose así (7").

$$
q=f_{1}\left[f_{2}\left(w, x, \alpha_{20}\right), m, \alpha_{10}\right]=(1+w-x) m \quad\left(7^{\prime \prime}\right)
$$

Insertando ( $\left.7^{\prime \prime}\right)$ en (16') se obtiene (16").

$$
\frac{p^{\prime}}{p}=f_{4}\left(p, w, q \cdot \alpha_{40}\right)=1-p+w-(1+w-x) m
$$

Ahora, se reemplaza en (A) los términos w', p' y m' por expresiones que no contengan derivadas. Para ello, se inserta en (A) las expresiones (s), (15'), $\left(16^{\prime \prime}\right)$ y $(\mathrm{E})\left(\mathrm{m}^{\prime} / \mathrm{m}=\gamma \mathrm{m}=1 \mathrm{O}^{-4}\right)$ lo cual genera (A'). Respecto a (B), en ésta se inserta ( $\left.7^{\prime \prime}\right)$, obteniéndose (B'). Juntando $\left(\mathrm{A}^{\prime}\right)$ y $\left(\mathrm{B}^{\prime}\right)$ con $\left(15^{\prime}\right),\left(16^{\prime \prime}\right)$ y $(\mathrm{E})$ a más de aplicar (s), se obtiene (A'-E').

$$
\begin{gathered}
g=\frac{(1+w-x) m-(w / p)}{m+w / p} \\
\frac{w^{\prime}}{w}=-0.25+0.35 x
\end{gathered}
$$




$$
\begin{gathered}
\frac{\boldsymbol{p}^{\prime}}{\boldsymbol{p}}=\mathbf{1}-\boldsymbol{p}+\boldsymbol{w}-(1+\boldsymbol{w}-\boldsymbol{x}) \boldsymbol{m} \quad\left(D^{\prime}\right) \\
\frac{\boldsymbol{m}^{\prime}}{\boldsymbol{m}}=10^{-4} \quad\left(E^{\prime}\right)
\end{gathered}
$$

Si bien en (A'-E') se presenta explícitamente la tasa de ganancia por su relevancia económica, puede ser útil para un estudio analítico de la dinámica del sistema insertar ( $\left.\mathrm{B}^{\prime}\right)$ en ( $\left.\mathrm{A}^{\prime}\right)$, de modo que surge un sistema en donde (A') y (B') se reemplazan por (AB').

$$
\frac{\boldsymbol{x}^{\prime}}{\boldsymbol{x}}=\frac{(1+\boldsymbol{w}-\boldsymbol{x}) \boldsymbol{m}^{2}+\left(\boldsymbol{p}-\boldsymbol{x}-10^{-4}\right) \boldsymbol{m}-(0.75+0.35 \boldsymbol{x})(\boldsymbol{w} / \boldsymbol{p})}{\boldsymbol{m}+\boldsymbol{w} / \boldsymbol{p}}
$$

29 Dicho procedimiento se hizo en Wolfram Mathematica 10 usando la función NDsolve. Para un trabajo futuro se estudiará el modelo con mayor rigurosidad analítica buscando bifurcaciones, así como usando coeficientes de Lyapunov.

30 La generalidad del modelo permite simular otros escenarios llamativos. Por ejemplo, si en el modelo $\left(A^{\prime}-D^{\prime}\right)$ se aumenta demasiado el efecto de los salarios sobre la inflación, surge inestabilidad a causa de una hiperinflación.

31 Aquí la palabra «ley» no debería interpretarse en términos «científicos», sino simplemente en términos de una posible tendencia del capitalismo.

32 Para una breve mención a las múltiples interpretaciones analíticas disponibles sobre la teoría del valor de Marx, se puede revisar el trabajo de Cajas-Guijarro (2015, p. 21).

\section{REFERENCIAS}

Basu, D., Chen, Y. y Oh, J. (2012). Class Struggle and economic flactuations: var Analysis of the postwar U. s. Economy. Documento de trabajo 2012-02, Universidad de Massachusetts, Estados Unidos. Recuperado de http://www.umass.edu/economics/publications/201202.pdf

Cajas-Guijarro, J. (2012, mayo). Relación entre tasa de explotación y desempleo, una breve extensión al modelo de Goodwin. Contribuciones a la economía. Recuperado de http://eumed.net/ce/2012/ jcg.html

Cajas-Guijarro, J. (2015). (2014). Clases sociales, desigualdad y subempleo en el capitalismo subdesarrollado. (Tesis de maestría). Recuperado de repositorio digital de la Flacso http://repositorio.flacsoandes.edu.ec/bitstream/10469/8146/2/TFLACSO-2015JWCG.pdf

Desai, M. (1978). Growth cycles and inflation in a model of the class Struggle. Journal of economic theory, $6(6), 527-545$.

Devine, J. (2005). Marx's law of capitalist accumulation revisited: Counteracting tendencies and internal dynamics. Recuperado de https://www.researchgate.net/publication/252291135_Marx's_Law_ of_Capitalist_Accumulation_Revisited_Counteracting_Tendencies_and_Internal_Dynamics

Flaschel, P. (2009). The macrodynamics of capitalism. Berlín, Alemania: Springer.

Goodwin, R. (1967). A growth cycle. En C. H. Feinstein (Ed.), Socialism, capitalism and economic growth. Essays presented to Maurice Dobb (54-58). Cambridge, Inglaterra: Cambridge University Press.

Goodwin, R. (1990). Chaotic economic dymanics. Oxford, Inglaterra: Oxford University Press.

Kalecki, M. (1954). Teoría de la dinámica económica. México DF, México: Editorial del Fondo de Cultura Económica. 
Kondratiev, N. D. (1935). The long waves in economic life. The Review of Economic Statistics, 17(6), 105-115. Mandel, E. (1979). Introducción al marxismo. Madrid, España: Akal Editor.

Mavroudeas, S. e Ioannides, A. (2011). Duration, intensity and productivity of labour and the distinction between absolute and relative surplus-value. Review of Political Economy, 23(3), 421-437.

Marx, K. (1844). The power of money. En K. Marx y F. Engels, Collected Works, (3), (322-326). Nueva York, Estados Unidos: International Publishers.

Marx, K. (1850). Circular del Comité Central a la Liga Comunista. Marxistis.org. Recuperado de http:// www.marxists.org/archive/marx/works/1847/communist-league/1850-ad1.htm

Marx, K. (1857-1858). Grundrisse: Elementos fundamentales para la crítica de la Economía Política, tomo II. México DF, México: Siglo xxi Editores.

Marx, K. (1867). El capital, tomo I: El proceso de producción del capital. México DF, México: Siglo XXI Editores

Marx, K. (1885). El capital, tomo II: El proceso de circulación del capital. México DF, México: Siglo XXI Editores.

Marx, K. (1894). El capital, tomo III: El proceso global de la producción capitalista. México DF, México: Siglo Xxi Editores.

Morishima, M. (1973). Marx's economics. A dual theory of value and growth. Cambridge, Inglaterra: Cambridge University Press.

Nietzsche, F. (1891). Así habló Zaratustra. Madrid, España: Alianza.

Noroña, S. y Cajas-Guijarro, J. (2014). Acumulación de capital, ejército industrial de reserva y su relación en la economía ecuatoriana. (Tesis de pregrado). Escuela Politécnica Nacional, Quito, Ecuador.

Nun, J. (1969). Superpoblación relativa, ejército industrial de reserva y masa marginal. Revista Latinoamericana de Sociología, 5(2), 180-225.

Phillips, A. (1958). The relation between unemployment and the rate of change of money wage rates in the United Kingdom, 1861-1957. Economica, 25(100), 283-299.

Quijano, A. (1973). Redefinición de la dependencia y proceso de marginalización social. En A. Quijano y F. Weffort (Eds.), Populismo, marginalización y dependencia (173-239). Recuperado de http:// anibalquijano.blogspot.com/2016/o2/1973-redefinicion-de-la-dependencia-y.html

Ricardo, D. (1821). Principios de Economía política y tributación. México DF, México: Fondo de Cultura Económica.

Shaikh, A. (1974). Laws of production and laws of algebra: the humbug production function. Review of Economics and Statistics, 56(1), 115-120.

Shaikh, Anwar (1990). Valor, acumulación y crisis. Bogotá, Colombia: Tercer Mundo Editores.

Shapiro, C. y Stiglitz, J. (1984). Equilibrium unemployment as a worker discipline device. The American Economic Review, 74(3), 433-444.

Solow, R. (1990). Goodwin's growth cycle: reminiscence and rumination. En J. Velupillai, (Ed.), Nonlinear Multisectoral Macrodynamics (31-41). Londres, Inglaterra: McMillan.

Sweezy, P. (1942). Teoría del desarrollo capitalista. México DF, México: Fondo de Cultura Económica.

Trotsky, L. (1939). Qué es el marxismo. Su moral y la nuestra. Madrid, España: Fundación Federico Engels. 